\title{
Application of Survival Tree Based on Texture Features Obtained through MRI of Patients with Brain Metastases from Breast Cancer
}

\author{
Asanao Shimokawa ${ }^{1, *}$, Yoshitaka Narita $^{2}$, Soichiro Shibui ${ }^{2}$ and Etsuo Miyaoka ${ }^{1}$ \\ ${ }^{1}$ Department of Mathematical Information, Tokyo University of Science, 1-3 Kagurazaka, Shinjuku-ku, Tokyo \\ 162-8601, Japan \\ ${ }^{2}$ Department of Neurosurgery and Neuro-Oncology, National Cancer Center Hospital, 5-1-1 Tsukiji, Chuo-ku, \\ Tokyo 104-0045, Japan
}

\begin{abstract}
The information obtained by magnetic resonance imaging (MRI) is considered to possess great potential for providing the prognosis of cancer patients, although not been established. The goal of this study was to evaluate the covariates of the texture patterns obtained from MRI scans of patients with breast cancer brain metastases, which influence the survival time prognosis. The data of forty patients were analyzed using 29 covariates. Twenty-six covariates, which are focused on the texture patterns, were calculated from the gray-level co-occurrence matrix and wavelet coefficients obtained by transform of preoperative T1-weighted MRI scans. The remaining three covariates were age, Karnofsky Performance Scale, and the indicator of whether solitary or multiple metastases were present. These covariates are commonly used as the prognostic factors in medical research. The tree structure prognosis models were constructed by applying the survival tree method to these covariates. The obtained survival trees separated the patients into two or three groups between which there was a statistically significant distance. For the purpose of comparison, Cox regression analyses were performed to determine which covariates showed significant predictive values. All the covariates selected in the Cox analysis and survival tree method were texture features only. In particular, the energy of the gray-level co-occurrence matrix and wavelet coefficients showed a high performance in tree structure analysis. From these results, we conclude that the features obtained from simple medical images can be used to estimate the prognosis of brain metastases patients.
\end{abstract}

Keywords: GLCM, wavelet transformation, recursive partitioning, binary tree, prognosis modeling, image analysis.

\section{INTRODUCTION}

In $10 \%$ of cancer patients, the probability of brain metastases occurring within a year is high and the survival time of these patients is approximately 1 year [1]. The typical prognostic factors that were determined by recursive partitioning analysis (RPA) are as follows: the Karnofsky Performance Scale (KPS), primary lesion (controlled vs. uncontrolled), age, and extracranial systemic metastases (present or absent) [2]. According to this classification, patients with a KPS score lower than 70 (RPA III) have the worst survival prognosis. The patients with the best survival prognosis have a KPS of at least 70 , are under 65 years of age, and have a controlled primary tumor (RPA I). This RPA classification is commonly used in clinical situations involving brain metastases. In a more recent research study, the effectiveness of the factors postoperative systemic therapy, controlled or uncontrolled extracranial malignancy, and postoperative KPS was shown from the aspect of early death [3]. Recently, the Graded Prognostic Assessment (GPA), which is a prognostic index for patients with brain metastases, was published [4]. This index, which comprises

*Address correspondence to this author at the Department of Mathematical Information, Tokyo University of Science, 1-3 Kagurazaka, Shinjuku-ku, Tokyo 162-8601, Japan; Tel: +81-3-3260-4271;

E-mail: a.shimokawa0226@gmail.com diagnosis-specific prognostic indices based on age, KPS, presence of extracranial systemic metastases, the number of brain metastases, and primary cancer site, can be used for the assessment of patients. Although these factors are used to estimate the survival time of cancer patients, there are many covariates that can potentially be used for this purpose. Recently, several studies on estimating the prognosis of patients with gliomas that used covariates obtained through magnetic resonance imaging (MRI) have been reported $[5,6]$. MRI is necessary for the diagnosis of and treatment decision for patients with brain metastases.

To identify novel prognostic factors, we used the survival tree method based on the classification and regression tree (CART) algorithm [7]. This method constructs the tree structure prognosis model by recursive partitioning. The constructed model classifies the patients into groups with similar covariates and prognosis. Although some authors used this algorithm to construct tree models for brain tumor patients [2-5], there are some differences in the goodness of split criterion function used in the algorithm. Because there is no gold standard for this criterion, we used criteria based on the results of comparative research using simulation [8]. 
The aim of this research study was to evaluate the covariates of texture patterns obtained from preoperative T1-weighted MRI scans of patients with breast cancer metastatic brain tumor. Texture is a feature that is typically used for identifying images; we used the gray-level co-occurrence matrix (GLCM) and wavelet transformation to obtain it. Ten statistical features, such as mean and variance, extracted from the GLCM were used as covariates. Sixteen features, such as energy and entropy, were used as covariates by wavelet transformation using the Daubechies D6 and D20 wavelets, which have been shown to be effective for classifying images [9-10]. Moreover, age, KPS score, and the indicator of whether solitary or multiple metastatic are present were used as the covariates.

This paper is orgnized as follows. The detail of the patients, the methods of image analysis, and the methods of statistical analysis are described in the next section. In Section 3, we show the experimental results. The discussion is described in Section 4.

\section{PATIENTS AND METHODS}

\subsection{Patients}

The data of forty patients with breast cancer brain metastases were analyzed in this study. All patients had at least a single metastasis, whose diameter was greater than $3 \mathrm{~cm}$. The patients underwent surgery as an initial treatment. The analysis in this study was approved by the local institutional review board of the national cancer center.

The patients underwent MRI examinations prior to surgery. The data were collected from the years 2000 to 2011. All patients were female, and their cancer onset age ranged from 37 to 79 years. They underwent surgery and standard radiation therapy. The observation period for analysis was defined as the time between surgery and death, when the date of death could not be confirmed, it was defined as the time until the final confirmation of survival. MRI data were acquired approximately 1 week before surgery.

All images were obtained using T1, T2, FLAIR (fluid-attenuated inversion recovery) and T1+Gd-DTPA (gadolinium with diethylenetriamine-pentaacetic acid) imaging. Among these images, we focused on T1 images, which were obtained using the following settings: axial plane, acquisition matrix (256-512) $\times(256-512), 19-27$ slices (in the case of one patient, only 10 slices).

\subsection{Image Analysis}

All images were skull stripped images and were processed by using the fast $2 \mathrm{D}$ watershed transform technique, semiautomatically [11]. The unnecessary regions to the study like eye, skull, and cerebral fluid, are removed from the images by this processing. As the result, the images that include the cerebral, midbrain, cerebellum, and oblongatal are obtained for the analysis. Two types of texture features were extracted from the skull stripped images: statistical texture features extracted from the GLCM of the image matrix and texture features extracted by using the wavelet transform of the image matrix.

$$
\text { Let } I=\left\{f\left(x_{1}, x_{2}\right) ; x_{1}=0,1, \cdots, N_{x_{1}}-1, x_{2}=0,1, \cdots, N_{x_{2}}-1\right\}
$$

be a two-dimensional image matrix, where $N_{x_{1}}$ and $N_{x_{2}}$ are the size of the image. Every element $f\left(x_{1}, x_{2}\right)$ have a gray level $\left\{g_{1}, g_{2}, \cdots g_{2^{b}}\right\}$, where $b$ represents quantization levels. In this study, we used a resolution (b) of 8. A GLCM is a $g_{2^{b}} \times g_{2^{b}}$ matrix whose elements represent the relationship between gray levels of two points in the image matrix I for a distance and direction vector $\boldsymbol{d}=\left(\Delta_{x_{1}}, \Delta_{x_{2}}\right)$, where $\Delta_{x_{1}}$ and $\Delta_{x_{2}}$ are fixed values. That is, each element of the GLCM $H=\left\{H\left(g_{q}, g_{r}, \boldsymbol{d}\right) ; q=1, \cdots 2^{b}, r=1, \cdots 2^{b}, \boldsymbol{d}=\left(\Delta_{x_{1}}, \Delta_{x_{2}}\right)\right\} \quad$ is given as follows:

$$
\begin{aligned}
& H\left(g_{q}, g_{r}, \boldsymbol{d}\right)=\operatorname{num}\left(f\left(x_{1}, x_{2}\right)=g_{q} \cap f\left(x_{1}+\Delta_{x_{1}}, x_{2}+\Delta_{x_{2}}\right)=\right. \\
& \left.g_{r} ; x_{1}=0, \cdots N_{x_{1}}-1, x_{2}=0, \cdots N_{x_{2}}-1\right) .
\end{aligned}
$$

By using $H\left(g_{q}, g_{r}, \boldsymbol{d}\right)$, we calcurate the follows:

$$
\begin{aligned}
& H_{m}\left(g_{q}, \boldsymbol{d}\right)=\sum_{r=1}^{2^{b}} H\left(g_{q}, g_{r}, \boldsymbol{d}\right), \\
& H_{\text {diff }}(i, \boldsymbol{d})=\sum_{q=1\left|g_{q}-g_{r}\right|=s}^{2^{b}} \sum_{r=1}^{2^{b}} H\left(g_{q}, g_{r}, \boldsymbol{d}\right)
\end{aligned}
$$

To calculate the GLCM for each image, we used $\boldsymbol{d}=(5,-5)$. Five features were obtained from the $H\left(g_{q}, g_{r}, \boldsymbol{d}\right)$ : contrast, correlation, energy, homogeneity, and entropy. Two features were obtained from the $H_{m}\left(g_{q}, \boldsymbol{d}\right)$ : mean and deviation. Three features were obtained from the $H_{\text {diff }}(i, \boldsymbol{d})$ : entropy, energy, and mean.

In this study, we have obtained one signal approximation coefficient and three wavelet coefficients 
Table 1: Summary Statistics of the Covariates

\begin{tabular}{|c|c|c|c|c|}
\hline Covariates & & Mean & Standard deviation & Cox $p$-value \\
\hline \multicolumn{5}{|l|}{ Commonly used covariates } \\
\hline KPS & 60 & 4 & 7.232 & 0.154 \\
\hline & 70 & 22 & & \\
\hline S/M metastatic & single & 29 & 0.452 & 0.573 \\
\hline & multiple & 11 & & \\
\hline \multicolumn{5}{|l|}{ GLCM } \\
\hline contrast & & 539.892 & 243.121 & 0.815 \\
\hline entropy & & 7.516 & 0.390 & 0.186 \\
\hline mean & & 85.904 & 16.827 & 0.923 \\
\hline deviation & & 21.089 & 4.552 & 0.382 \\
\hline entropy of dif. & & 3.555 & 0.225 & 0.845 \\
\hline mean of dif. & & 0.046 & 0.011 & 0.982 \\
\hline energy of dif. & & 13.981 & 3.328 & 0.778 \\
\hline \multicolumn{5}{|l|}{ wavelet transform } \\
\hline energy of app. (D6) & & 8252.027 & 3481.806 & 0.643 \\
\hline energy of hor. (D6) & & 14.940 & 9.863 & 0.026 \\
\hline entropy of app. (D6) & & 3.583 & 0.181 & 0.113 \\
\hline entropy of hor. (D6) & & 2.793 & 0.221 & 0.046 \\
\hline entropy of ver. (D6) & & 2.789 & 0.215 & 0.051 \\
\hline entropy of dia. (D6) & & 2.644 & 0.171 & 0.051 \\
\hline entropy of app. (D20) & & 3.584 & 0.181 & 0.112 \\
\hline entropy of hor. (D20) & & 2.901 & 0.205 & 0.044 \\
\hline entropy of ver. (D20) & & 2.890 & 0.183 & 0.042 \\
\hline entropy of dia. (D20) & & 2.789 & 0.151 & 0.062 \\
\hline
\end{tabular}

from the image matrix by single-level two-dimensional wavelet transformation. The Daubechies D6 and D20 wavelets were selected for use in wavelet transformation, following the method used in a previous study [10]. Sixteen features were obtained by wavelet transformation: energy and entropy from one signal approximation coefficient and three wavelet coefficients. All features were extracted from each slice, and the mean values were defined as covariates. The details of the extraction methods are described in [12].

\subsection{Statistical Analysis}

All patients were classified according to the covariates, which were age, KPS score, solitary or 
multiple brain metastases indicator, and the 26 texture features described in the previous section. The summary statistics of these covariates are shown in Table 1. To identify effective prognostic factors and seek their splitting point for grouping, we used the survival tree method. The survival tree method is a statistical tool that constructs a tree model according to the goodness of splits in terms of covariates and prognosis. The resulting model differs according to the splitting criteria, which were proposed in various papers [13-20]. We performed the comparative study of the splitting criteria by using simulations as previous research [8]. Based on the research, three patterns of splitting criteria were used in this study: exponential log-likelihood loss (EL), deviance residual under the proportional hazard model (PD), and the logrank test statistic (LR).

Let $L_{t}=\left\{\left(x_{i}, \delta_{i}, z_{i}\right) ; i=1,2, \cdots, N_{t}\right\}$ be the set of samples in an arbitrary internal node $t$, where $x_{i}$ is the observation time, $\delta_{i}$ is the censoring indicator, which is 1 if the observation is an event and is 0 if the observation is censored, and $z_{i}$ is the covariate vector of sample $i$. Then, the EL criterion in each internal node $t$ is given as follows:

$$
R(t)=\sum_{i \in L_{t}} \delta_{i}-\sum_{i \in L_{t}} \delta_{i} \log \left(\frac{\sum_{i \in L_{t}} \delta_{i}}{\sum_{i \in L_{t}} x_{i}}\right) .
$$

The PD criterion is given by

$$
R(t)=\sum_{i \in L_{t}} 2\left[\delta_{i} \log \left(\frac{\delta_{i}}{\widehat{\Lambda}_{0}\left(x_{i}\right) \hat{\theta}_{t}}\right)-\left(\delta_{i}-\widehat{\Lambda}_{0}\left(x_{i}\right) \hat{\theta}_{t}\right)\right]
$$

where $\hat{\Lambda}_{0}\left(x_{i}\right)$ represents the Nelson-Aalen estimator at $x_{i}$, and the $\hat{\theta}_{t}$ is given by

$\widehat{\theta}_{t}=\frac{\sum_{i \in L_{t}} \delta_{i}}{\sum_{i \in L_{t}} \hat{\Lambda}_{0}\left(x_{i}\right)}$

The split that minimizes $R(t)$ is selected in each internal node for constructing the child nodes about these criteria. The LR criterion is given by

$$
G(t)=\frac{\sum_{i \in L_{t}}\left[d_{L i}-E\left(D_{L i}\right)\right]}{\sum_{i \in L_{t}} \operatorname{Var}\left(D_{L i}\right)}
$$

where the $E\left(D_{L i}\right)$ and $\operatorname{Var}\left(D_{L i}\right)$ represent the expectation and variance of the number of events in the left child node $t_{L}$, respectively. $d_{L i}$ is the number of events at $x_{i}$ in the node $t_{L}$. The split that maximizes $G(t)$ is selected in each internal node.

In this study, each setting for constructing survival trees was defined as follows. The minimum number of events in nodes as the stop condition of the splitting step was set to 5 or less and the optimal size of the trees was determined based on the 10 -fold crossvalidation results [7].

Kaplan-Meier curves were plotted for each group of patients, categorized by the terminal nodes in the tree model. Moreover, logrank tests were performed to compare the relationship between the groups.

To determine which covariates showed significant predictive values, individual Cox regression analyses were also performed separately [21]. Moreover, the covariates that were considered to be useful for estimating a prognosis were included in a second Cox analysis.

All the image and statistical analyses were performed in MATLAB (Version R2013a, Mathworks Inc., Natick, MA, USA).

\section{RESULTS}

The overall Kaplan-Meier curve for all patients is displayed in Figure 1. The median survival time was 414 days, while the five-year survival rate was only $9.2 \%$. The results of the Cox regression analysis of

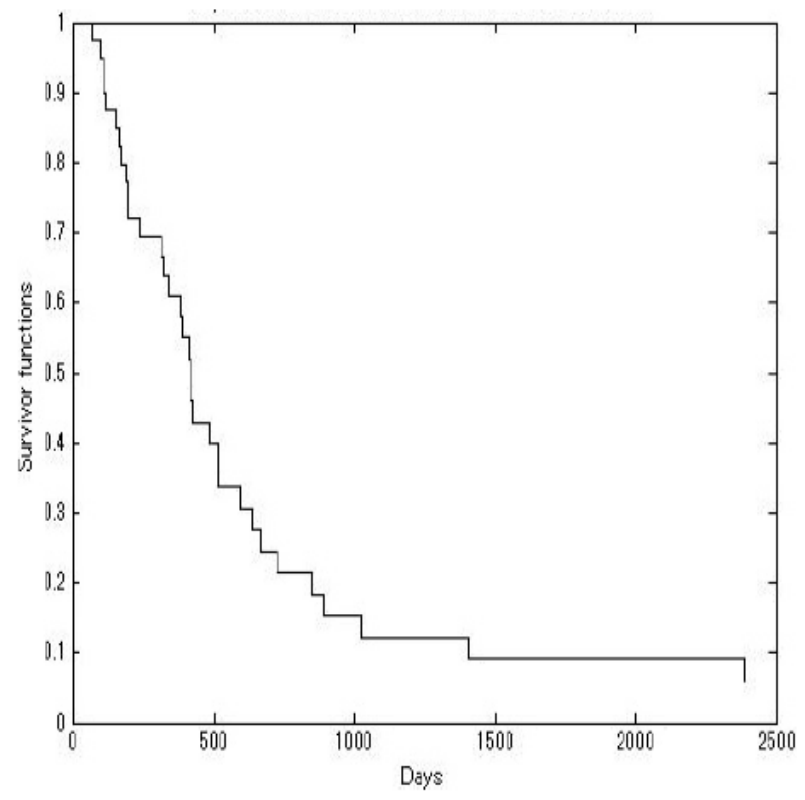

Figure 1: Kaplan-Meier survival curve for all patients. 
individual covariates are shown in Table 1. According to the $p$-values, which were calculated by a Wald test, the covariates commonly used in medical research are not statistically significant for prognosis nor are the covariates extracted from the GLCM. However, the five covariates obtained by wavelet transformation showed $p$-values lower than 0.05. By stepwise selection with an entry parameter of $0.05-1.0$ and stay parameter of $0.02-0.09$, the energy of the horizontal and vertical wavelet coefficients (D20) were included in the Cox regression model, with a $p$-value of 0.013 and 0.002 for the horizontal and vertical coefficients, respectively. If the stay parameter is set to $0.09-0.20$, then the entropy of the vertical wavelet coefficients (D6) was additionally included in the Cox regression model, with a $p$-value of 0.095 .

Survival trees were constructed using the 29 covariates described in Table 1. First, we used the result of the Cox regression analysis of individual covariates to reduce the number of covariates which are used in the survival tree analysis. In this study, the texture features that the $p$-value is greater than 0.5 are not used in the analysis. As the result, survival trees were constructed using the 20 covariates. The EL, PD, and LR criteria yielded different results. For the EL criterion, the obtained tree structure is shown in Figure 2. The circle and squares in the figure represent the internal nodes and terminal nodes, respectively. The values in the shapes represent the number of samples in the node, and the values in parentheses represent the number of events. The tree has only one split and two terminal nodes; the covariate used in the tree was the energy extracted from the GLCM. The KaplanMeier survival functions for each terminal node $\left(t_{1}, t_{2}\right)$ are shown in Figure 3. The patients in node $t_{1}$, which includes patients having a lower GLCM energy, had a higher risk of death than those in node $t_{2}$. The median survival of the patients in node $t_{1}$ was 323 days, and their five-year survival rate was $0 \%$. However, the median survival of patients in node $t_{2}$ was 593 days, and their five-year survival rate was $12.6 \%$. The $p$ -

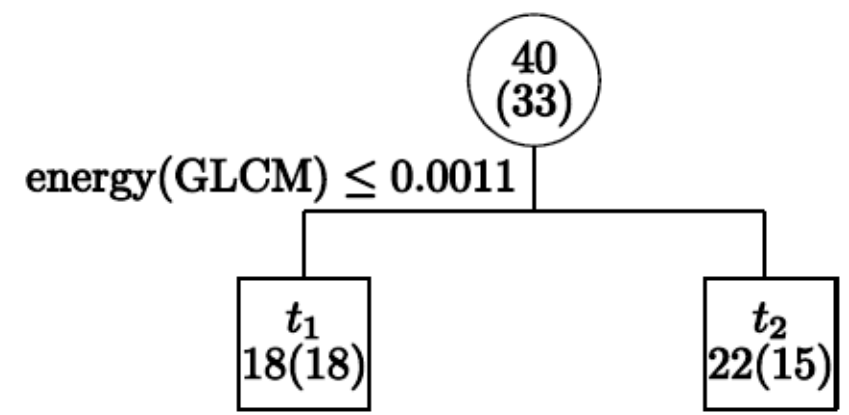

Figure 2: Survival tree by EL criterion. value of the logrank test for nodes $t_{1}$ and $t_{2}$ was 0.0091 , showing that a statistically significant difference in prognosis existed between the two groups of patients.

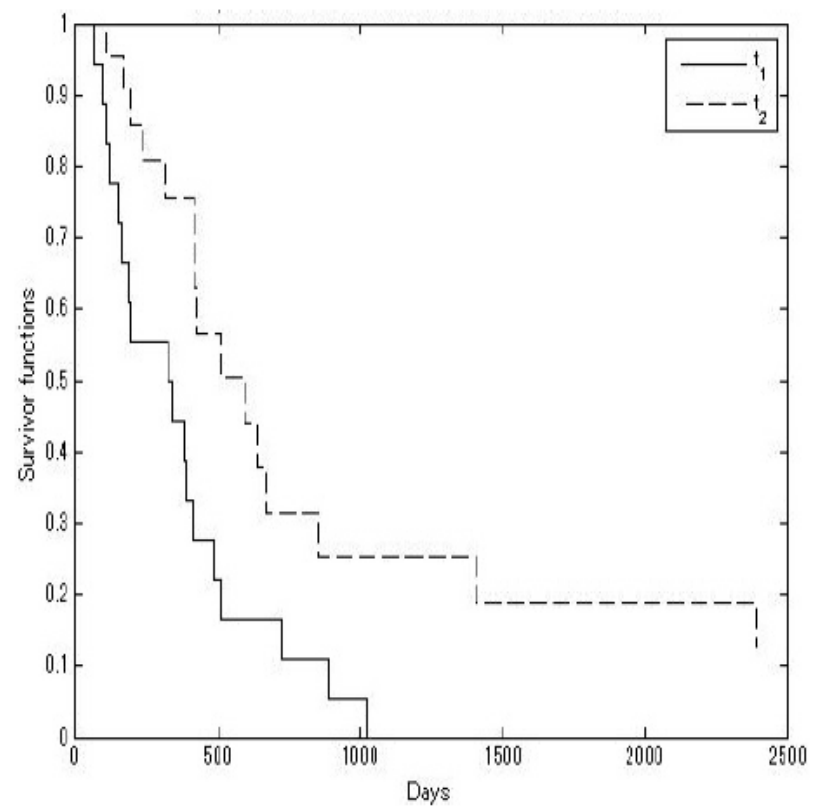

Figure 3: Kaplan-Meier survival curves for the terminal nodes in Figure 2.

For the PD criterion, the obtained tree structure is given in Figure 4. The tree has two splits and three terminal nodes. The covariates used in the tree were the energy of the horizontal wavelet coefficient (D20) and the energy of the diagonal wavelet coefficient (D6). The Kaplan-Meier survival functions for each terminal node $\left(t_{1}, t_{3}\right)$ are shown in Figure 5 . The patients in node $t_{3}$, which includes patients whose energy of horizontal wavelet coefficient (D20) was higher, had the highest risk of death; those in node $t_{2}$, which includes patients whose energy of horizontal wavelet coefficient (D20) was lower and energy of diagonal wavelet coefficient (D6) was higher, had the lowest risk of death. The median survival time of the patients

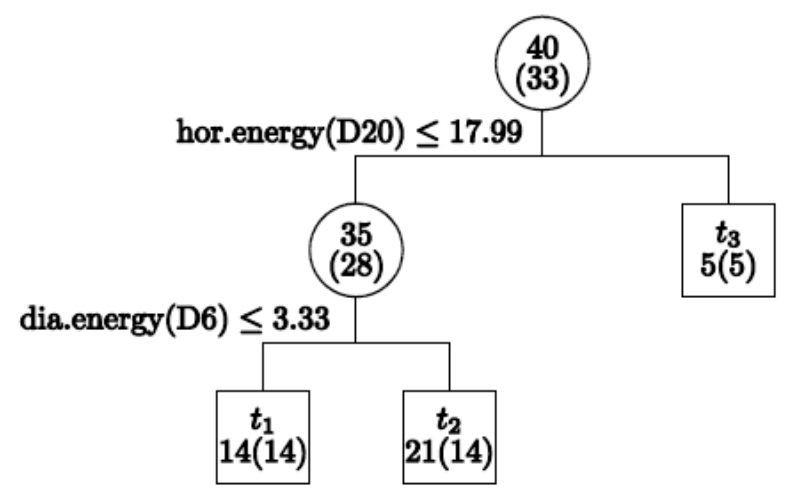

Figure 4: Survival tree by PD criterion. 
included in nodes $t_{1}, t_{2}$, and $t_{3}$ was 323 days, 672 days, and 114 days, respectively. The five-year survival rate of patients included in these nodes was $0 \%$, $12.89 \%$, and $0 \%$, respectively. The $p$-values of the logrank tests for each combination of nodes showed that statistically significant differences in prognosis existed between the groups: $p$-value $t_{1}-t_{2}=0.0003 ; p$ value $\left(t_{2}-t_{3}\right)<.0001 ; p$-value $\left(t_{3}-t_{1}\right)=0.0037$.

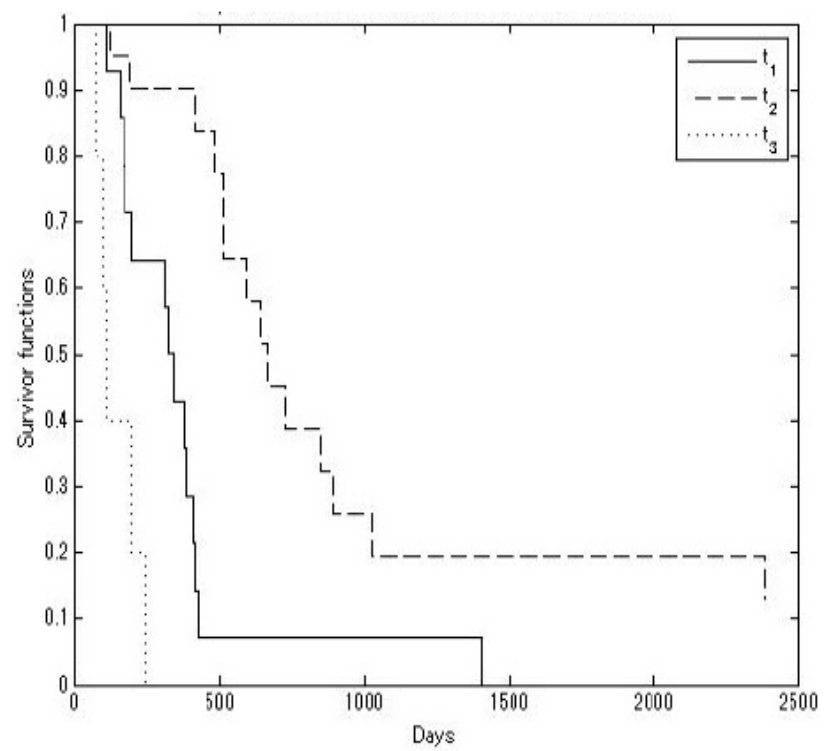

Figure 5: Kaplan-Meier survival curves for the terminal nodes in Figure 4.

The tree structure obtained by the LR criterion is given in Figure 6 . The tree has one split and two terminal nodes; the covariate used in the tree was the energy of the horizontal wavelet coefficient (D6). The Kaplan-Meier survival functions for each terminal node $\left(t_{1}, t_{2}\right)$ are shown in Figure 7. The patients in node $t_{1}$, which includes the patients whose energy of horizontal wavelet coefficient (D6) was lower, had a lower risk of death than those in node $t_{2}$. The median survival time of the patients in node $t_{1}$ was 431 days, and their fiveyear survival rate was $7.02 \%$. In contrast, the median survival time of the patients in node $t_{2}$ was 114 days, and their five-year survival rate was $0 \%$. The $p$-value of

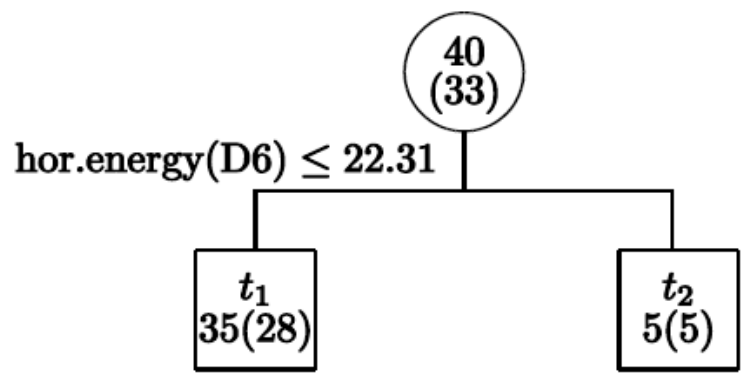

Figure 6: Survival tree by LR criterion. the logrank test was $<.0001$, showing that a statistically significant difference in prognosis existed between the two groups.

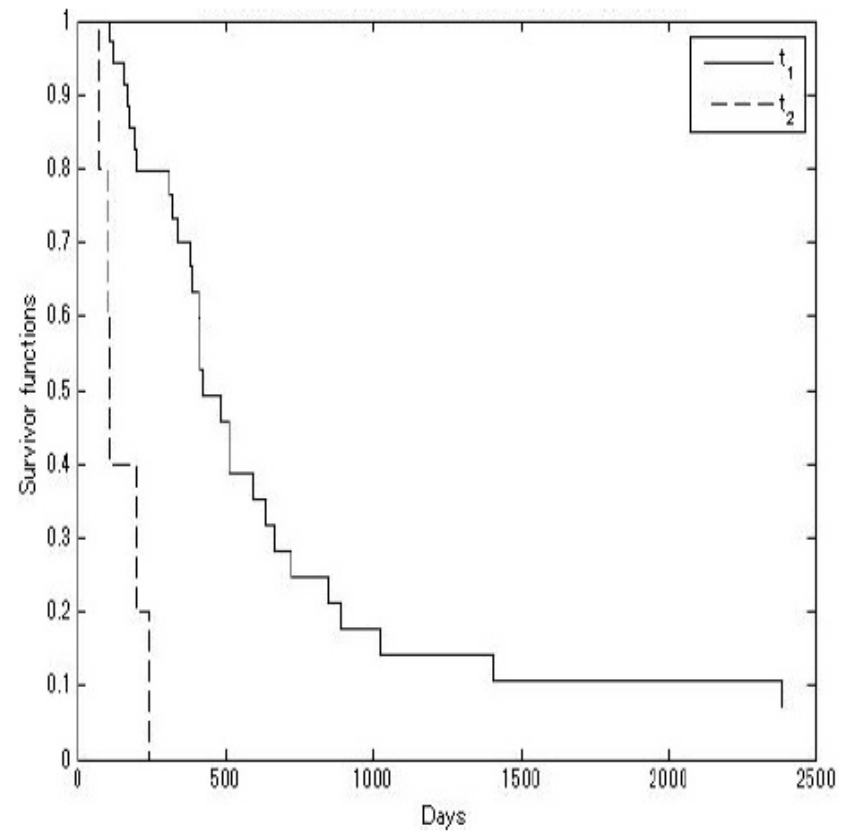

Figure 7: Kaplan-Meier survival curves for the terminal nodes in Figure 6.

\section{DISCUSSION}

The prognosis of patients with brain metastases depends on various factors. In the previous studies, the KPS, primary lesion, age, extracranial systemic metastases, controlled or uncontrolled extra-cranial malignancy, postoperative KPS, the number of brain metastases, and primary cancer site have been considered as effective to estimate the prognosis of brain metastases patients $[2,3,4]$. We focused on the more objective features obtained from MRI and searched the factors that affect survival. The texture patterns of images, which were obtained by GLCM and wavelet transformation, were proven to be useful in this study. The Cox proportional hazard model and the survival tree methods were used to evaluate the covariates. The results showed that the objective features obtained from simple medical images can be used effectively to obtain a prognosis.

To identify which covariates show significant predictive values, 26 covariates relevant to texture pattern and three covariates that are commonly used in medical research for the purpose of prognosis were investigated using Cox regression analyses. Three commonly used covariates, age, KPS, and solitary or multiple brain metastases indicator, did not yield statistically significant results for prognosis in this 
study. As regards the texture patterns, 10 features obtained from GLCM also did not yield statistically significant results. In contrast, some of the 16 features obtained by wavelet transformation yielded significant results. Moreover, the model that includes two or three features obtained by wavelet transformation was selected as the optimal regression model by the stepwise selection method.

Next, we performed an additional analysis using the survival tree method, which is assumed to be a nonparametric model. The survival tree method based on the CART algorithm segregates patients into groups with similar covariates and prognosis. There are various proposed criterion functions for the splitting step in the algorithm [13-20]. The modified wilcoxon criterion has been used as the splitting criterion in the study of [2]. On the other hand, the Log-rank statistics has been used in the study of [5]. However, it is known that the resulting model differs according to the splitting criteria. Therefore, we used three criteria based on previous simulation results. The trees obtained based on each criterion show different results. The covariates selected in the final trees are texture features obtained from MRI images: energy extracted from GLCM, energy of horizontal wavelet coefficient (D20), energy of diagonal wavelet coefficient (D6), and energy of horizontal wavelet coefficient (D6). The Kaplan-Meier survival curves for each group were well separated, and the $p$-values of the logrank tests show statistically significant differences between the groups.

Although age and KPS, in particular, are considered effective prognostic factors for brain metastases patients according to a previous large scale survey, we could not find evidence that these covariates are effective for estimating the survival time of patients with breast cancer brain metastases. In contrast, texture features obtained from MRI were determined to be effective prognosis factors in Cox analysis and survival tree analysis. In particular, the energy of GLCM and wavelet coefficients showed high-level performance in tree structure analysis.

Finally, the Cox proportional hazard model is an effective, powerful, and widely used tool in the analysis of survival data. However, this model requires several assumptions and its interpretation is not easy when this model includes many covariates. The survival tree method, which recursively partitions the data into groups with similar covariates and prognosis, can determine the relationship between covariates and hazards easily. Moreover, a new patient can easily be incorporated into the model. In the previous comparative study [22], it is shown that the survival tree can give the another aspect of the data compared to the Cox model. In this study, we demonstrated the application of the survival tree method and the effectiveness of using this method for survival analysis. According to the results obtained using the Cox model and the survival tree method, texture patterns obtained by simple preoperative MRI can be considered valid prognosis factors for patients with brain metastases from breast cancer.

\section{REFERENCES}

[1] Narita $Y$, Shibui S. Strategy of surgery and radiation therapy for brain metastases. Int J ClinOncol 2009; 14(4): 275-280. http://dx.doi.org/10.1007/s10147-009-0917-0

[2] Gaspar L, Scott C, Rotman M, et al. Recursive partitioning analysis (RPA) of prognostic factors in three Radiation Therapy Oncology Group (RTOG) brain metastases trials. Int J Radiat Oncol Biol Phys 1997; 37(4): 745-751. http://dx.doi.org/10.1016/S0360-3016(96)00619-0

[3] Arita H, Narita $\mathrm{Y}$, Miyakita $\mathrm{Y}$, et al. Risk factors for early death after surgery in patients with brain metastases: reevaluation of the indications for and role of surgery. $J$ Neurooncol 2014; 116: 145-152.

http://dx.doi.org/10.1007/s11060-013-1273-5

[4] Sperduto PW, Kased N, Roberge D, et al. Summary Report on the Graded Prognostic Assessment: An Accurate and Facile Diagnosis-Specific Tool to Estimate Survival for Patients With Brain Metastases. J Clin Oncol 2012; 30: 419425.

http://dx.doi.org/10.1200/JCO.2011.38.0527

[5] Li X, Jin H, Lu Y, et al. J. Identification of MRI and ${ }^{1} \mathrm{H}$ MRSI parameters that may predict survival for patients with malignant gliomas. NMR Biomed 2004; 17(1): 10-20. http://dx.doi.org/10.1002/nbm.858

[6] Sanz-Requena R, Revert-Ventura A, Martí-Bonmatí L, Alverich-BayarriÁ, García a-MartíG. Quantitative MR perfusion parameters related to survival time in high-grade gliomas. Eur Radiol 2013; 23: 3456-3465. http://dx.doi.org/10.1007/s00330-013-2967-y

[7] Breiman L, Friedman JH, Olshen RA, Stone C. Classification and Regression Trees. Wadsworth, California 1984.

[8] Shimokawa A, Kawasaki Y, Miyaoka E. Comparison of the splitting criterions of survival tree. Proceedings of the 27th Symposium of Japanese Society of Computational Statistics 2013 (in Japanese): Nov 15-16; Kumamoto, Japan: p. 217220.

[9] Daubechies I. Orthonormal Bases of Compactly Supported Wavelets. Commun Pure Appl Math 1988; 41: 909-996. http://dx.doi.org/10.1002/cpa.3160410705

[10] Laine A, Fan J. Texture Classification by Wavelet Packet Signatures. IEEE Trans Pattern Anal Mach Intell 1993; 15: 1186-1191.

http://dx.doi.org/10.1109/34.244679

[11] Horst KH, Heinz OP. The Skull Stripping Problem in MRI Solved by a Single 3D Watershed Transform. Med Image Comput Comput Assist Interv 2000; 1935: 134-143. http://link.springer.com/chapter/10.1007\%2F978-3-54040899-4_14

[12] Shimokawa A, Miyaoka E. Application of genetic algorithm for classification of medical images. Adv Appl Stat 2012; 29(1): 1-31. http://www.pphmj.com/abstract/7063.htm 
[13] Gordon L, Olshen RA.Tree-Structured Survival Analysis. Cancer Treat Rep1985; 69(10): 1065-1069. http://test.europepmc.org/abstract/MED/4042086/reload=2;js essionid=17D76A53C8A374608ABEE95C001879EA

[14] Ciampi A, Hogg SA, Mckinney S, Thiffault J. RECPAM: A Computer Program for Recursive Partition and Amalgamation for Censored Survival Data and Other Situations Frequently Occurring in Biostatistics. I Methods and Program Features. Comput Methods Programs Biomed 1988; 26(3): 239-256. http://dx.doi.org/10.1016/0169-2607(88)90004-1

[15] Segal MR. Regression Trees for Censored Data. Biometrics 1988; 44: 35-47. http://dx.doi.org/10.2307/2531894

[16] Davis RB, Anderson JR. Exponential Survival Trees. Stat Med 1989; 8: 947-961. http://dx.doi.org/10.1002/sim.4780080806

[17] Therneau TM, Grambsch PM, Fleming TR. Martingale-Based Residual for Survival Models. Biometrika 1990; 77: 147-160. http://dx.doi.org/10.1093/biomet/77.1.147
[18] Leblanc M, Crowley J. Relative Risk Trees for Censored Survival Data. Biometrics1992; 48: 411-425. http://dx.doi.org/10.2307/2532300

[19] Zhang HP. Splitting Criteria in Survival Trees. In: Seeber G Francis BJ, Hatzinger R, Steckel-Berger G, editors. Proceedings of the 10th International Workshop on Statistical Modeling; 1995: July 10-14; Innsbruck, Australia: p. 305-314. http://www.springer.com/mathematics/probability/book/978-0387-94565-1

[20] Keles S, Segal MR. Residual-based tree-structured survival analysis. Stat Med 2002; 21(2): 313-326. http://dx.doi.org/10.1002/sim.981

[21] Cox DR. Regression Models and Life-Tables. J R Stat Soc Series B Stat Methodol 1972; 34: 187-220.

[22] Ciampi A, Thiffault J, Nakache JP, Asselain B. Stratification by stepwise regression, correspondence analysis and recursive partition: A comparison of three methods of analysis for survival data with covariates. Comput Stat Data Anal 1986; 4(3): 185-204. http://dx.doi.org/10.1016/0167-9473(86)90033-2

Received on 21-04-2014

http://dx.doi.org/10.6000/1929-6029.2014.03.04.2

(c) 2014 Shimokawa et al.; Licensee Lifescience Global.

This is an open access article licensed under the terms of the Creative Commons Attribution Non-Commercial License (http://creativecommons.org/licenses/by-nc/3.0/) which permits unrestricted, non-commercial use, distribution and reproduction in any medium, provided the work is properly cited. 